\title{
Suppression of nuclear factor erythroid-2-related factor 2-mediated antioxidative defense in the lung injury induced by chronic exposure to methamphetamine in rats
}

\author{
YANG BAI $^{1}$, YUN WANG $^{1}$, MING LIU $^{2}$, YU-HAN GU $^{1}$, BIN JIANG $^{3}$, XU WU ${ }^{4}$ and HUAI-LIANG WANG ${ }^{1,5}$ \\ ${ }^{1}$ Department of Clinical Pharmacology, School of Pharmacy, China Medical University, Shenyang, \\ Liaoning 110122; ${ }^{2}$ Department of Drug Control, China Criminal Police University, Shenyang, Liaoning 110035; \\ ${ }^{3}$ Department of Cardiovascular Ultrasound, The First Hospital, China Medical University, Shenyang, \\ Liaoning 110001; ${ }^{4}$ Department of Forensic Pathology, School of Forensic Medicine, China Medical University, \\ Shenyang, Liaoning 110122; ${ }^{5}$ National Key Subject, Institute of Respiratory Diseases and Institute of \\ Cardiovascular Diseases, China Medical University, Shenyang, Liaoning 110001, P.R. China
}

Received January 5, 2016; Accepted February 10, 2017

DOI: $10.3892 / \mathrm{mmr} .2017 .6356$

\begin{abstract}
The imbalance between oxidative stress and antioxidant defense is important in the pathogenesis of lung diseases. Nuclear factor erythroid-2-related factor 2 (Nrf2) is a key transcriptional factor that regulates the antioxidant response. The purpose of the present study was to investigate whether Nrf2-mediated antioxidative defense is involved in methamphetamine (MA)-induced lung injury in rats. Following establishment of chronic MA toxicity in rats, Doppler ultrasonic detection was used to measure the changes of physiological indexes, followed by hematoxylin and eosin staining, ELISA and western blot analysis. MA was demonstrated to increase the heart rate and peak blood flow velocity of pulmonary arterial valves and to decrease the survival rate of rats, and resulted in lung injury characterized by perivascular exudates, airspace edema, slight hemorrhage and inflammatory cell infiltration. MA significantly inhibited the expression of nuclear Nrf2 protein and its target genes (glutamate-cysteine ligase catalytic subunit $\mathrm{C}$ and heme oxygenase-1), and dose-dependently reduced glutathione (GSH) levels and the ratio of GSH/oxidized glutathione, accompanied by increases in reactive oxygen species (ROS) levels in rat lungs. Linear regression analysis revealed that there was a positive correlation between lung ROS level and lung injury indexes. These findings suggested that chronic exposure to MA led to lung injury by suppression of Nrf2-mediated antioxidative defense,
\end{abstract}

Correspondence to: Dr Yun Wang, Department of Clinical Pharmacology, School of Pharmacy, China Medical University, 77 Puhe Road, Shenyang, Liaoning 110122, P.R. China E-mail: ywang28@cmu.edu.cn

Key words: methamphetamine, nuclear factor erythroid-2-related factor 2, oxidative stress, reactive oxygen species, glutamate-cysteine ligase catalytic subunit $\mathrm{C}$, heme oxygenase-1, glutathione suggesting that Nrf2 may be an important therapeutic target for MA-induced chronic lung toxicity.

\section{Introduction}

Methamphetamine (MA) is an addictive drug that is abused globally (1). MA abuse significantly increases the risk of developing pulmonary arterial hypertension (PAH) $(2,3)$. Oxidative stress, which occurs in diseased lungs and is associated with $\mathrm{PAH}$, is thought to be responsible for the progression of cardiopulmonary changes $(4,5)$. Reactive oxygen species (ROS) may serve as signaling molecules to mediate multiple cell functions, and have been implicated in the pathogenesis of many diseases $(6,7)$. ROS formation additionally controls inducible expression of chemokines and other inflammatory genes in response to infection, implying a causal relationship between increased ROS production and lung injury $(8,9)$. MA abuse has previously been demonstrated to induce pulmonary toxicity in rats (10). Therefore, it has been hypothesized that methamphetamine may have the capacity to generate oxidative damage in lung tissues by inducing the formation of free radicals, including ROS.

Oxidative stress interferes with the expression of genes and transcriptional factors, including nuclear factor erythroid-2-related factor 2 (Nrf2)-dependent antioxidant response element (ARE) (11). It has been reported that low levels of ROS may induce Nrf2 activation (12). Nrf2 belongs to a small family of transcription factors containing a unique basic-leucine-zipper motif, the cap-n-collar (CNC) family (13). $\mathrm{Nrf} 2$ is a transcription factor that is involved in cellular defense against oxidative stress and electrophilic insults (14). $\mathrm{Nrf} 2$ is located in the cytoplasm as an inactive form associated with its repressor protein, Kelch-like ECH associating protein 1 (Keap1) (15). Oxidation of redox-sensitive cysteines in Keap1 during oxidative stress allows Nrf2 to dissociate from Keap1 and translocate to the nucleus, where it heterodimerizes with other proteins and binds to ARE, an enhancer sequence that regulates the transcription of cytoprotective 
enzymes including glutamate-cysteine ligase catalytic subunit C (GCLC), glutathione S-transferase (GST) and heme oxygenase-1 (HO-1) $(15,16)$.

Nrf2 induces the expression of several oxidant-signaling proteins that affect particular cellular functions, including autophagy, inflammation, apoptosis and mitochondrial biogenesis, by controlling the transcription of several drug metabolizing enzymes, transporters, cellular reducing equivalents including reduced glutathione (GSH) and nicotinamide adenine dinucleotide phosphate, and proteasomes (17). Multiple enzymes regulated by Nrf2 are essential in the pathogenesis of cardiovascular diseases, including atherosclerosis, ischemia-reperfusion injury and hypertension (18). However, little evidence has been provided concerning whether Nrf2 and its regulated enzymes are related to MA-induced lung injury. The present study was designed to investigate the function of Nrf2 and whether the suppression of Nrf2-mediated antioxidative defense is associated with lung injury induced by chronic exposure to MA in rats.

\section{Materials and methods}

Drug. MA was obtained from China Criminal Police University (Shenyang, China). The identity and purity of MA were determined using a Bio-Rad REMEDi HS system (Bio-Rad Laboratories, Inc., Hercules, CA, USA) and confirmed by liquid chromatography-mass spectrometry-mass spectrometry (Department of Drug Control, China Criminal Police University). MA was dissolved in $0.9 \%$ sterile saline at $4 \mathrm{mg} / \mathrm{ml}$ for drug administration.

Animals and procedures. All procedures were approved by the Institutional AnimalCare and Use Committee of China Medical University (Shenyang, China). Male Wistar rats ( $\mathrm{n}=30$; age, 7 weeks; weight, $180 \pm 10 \mathrm{~g}$ ) from the Animal Resource Center, China Medical University (Shenyang, China; certificate no. Liaoning 034 ) were divided into 3 groups ( $\mathrm{n}=10 /$ group): $0.9 \%$ saline (control), $5 \mathrm{mg} / \mathrm{kg} \mathrm{MA} \mathrm{(M5),} \mathrm{and} 10 \mathrm{mg} / \mathrm{kg} \mathrm{MA} \mathrm{(M10).}$ Rats were administrated twice daily for 5 weeks. They were maintained in standard conditions (at $18-22^{\circ} \mathrm{C}, 50 \%$ humidity) throughout the experimental period and were given ad libitum access to food and water in an alternating 12-h light/dark cycle over a period of 5 weeks. The procedure of establishment of the rat chronic lung injury models was in accordance with previous studies $(10,19)$.

Doppler ultrasonic detection of physiological indexes. The abdomen hair of rats was thoroughly clipped and shaved immediately following intraperitoneal anesthesia with $3 \%$ pentobarbital sodium (45 mg/kg; Sigma-Aldrich; Merck KGaA, Darmstadt, Germany) to achieve full contact of the probe with the skin in the region concerned. The rats were examined in the supine position. Acoustic gel (Skintact; Leonard Lang $\mathrm{GmbH}$, Innsbruck, Austria) was applied to hold the animal dander andimprove the resolution of the ultrasound. A Philips IE33 cardiovascular ultrasound (Philips Healthcare, Andover, MA, USA) with a linear transducer probe S5-1 was used. The frequency of $4.5 \mathrm{MHz}$ and one focal zone set at a depth of $0.5-2 \mathrm{~cm}$ were used to achieve detailed imaging of the rats.
Lung wet-to-dry weight (W/D) ratio. In the present study, lung W/D weight ratio was calculated to assess tissue edema. Rats were euthanized 5 weeks following MA administration. The left upper lobes were isolated, blotted dry and weighed to obtain the 'wet' weight. Following this, the left upper lobes were placed into an oven at $80^{\circ} \mathrm{C}$ for $48 \mathrm{~h}$ to obtain the 'dry' weight. The ratio of the wet lung to dry lung was calculated to quantify the magnitude of pulmonary edema.

Histological evaluation. The left lower lung lobes were isolated and fixed with $4 \%$ paraformaldehyde for $24 \mathrm{~h}$. Following washing 3 times with $0.1 \mathrm{M}$ PBS (pH 7.2), the fixed tissues were prepared for paraffin embedding and then cut into $5 \mu \mathrm{m}$ thick sections. To examine the inflammatory aspects of the lung, the sections were processed for hematoxylin and eosin staining. The stained sections were imaged under a Olympus BX51 fluorescence microscope (Tokyo, Japan) at an original magnification of $x 200$. Six sections from each sample were evaluated and scored independently by two members of the laboratory trained in histological assessment and use of the scoring system. Different lobes were examined for the following features: Interstitial edema, hemorrhage and inflammatory cell infiltration. Each feature received a score of 0 , no injury; 1 , minimal injury; 2 , moderate injury; or 3 , severe injury. This was totaled for a given lobe's score, and 3 lobes per rat (2-3 rats per group) were averaged to generate a score, giving a minimum score of 0 and a maximum of 9 (20).

Western blot assay. The right lungs were stored in liquid nitrogen at $-80^{\circ} \mathrm{C}$ until analysis. Lung tissues were homogenized in radioimmunoprecipitation lysis buffer (cat. no. P0013B; Beyotime Institute of Biotechnology, Haimen, China) containing protease inhibitor phenylmethylsulfonyl fluoride (cat. no. ST506; Beyotime Institute of Biotechnology) on ice, and protein concentrations were determined using Bradford reagent (Bio-Rad Laboratories, Inc.). Nuclear and cytoplasmic fractions were extracted using aprotein extraction reagent (cat. no. P0027; Beyotime Institute of Biotechnology). The protein concentrations were determined using a bicinchoninic acid protein assay kit (Beyotime Institute of Biotechnology) prior to storage at $-80^{\circ} \mathrm{C}$. Protein samples $(80 \mu \mathrm{g})$ were separated by $10 \%$ SDS-PAGE and subsequently transferred onto a polyvinylidene fluoride membrane (EMD Millipore, Billerica, MA, USA). Following blocking of the nonspecific site with 5\% non-fat dry milk for $1 \mathrm{~h}$ at room temperature, the membrane was incubated with primary rabbit anti-Nrf2 (1:600 dilution; cat. no. 16396-1-AP; Proteintech Group, Inc., Chicago, IL, USA), anti-GCLC (1:200 dilution; cat. no. bs-8402R; Beijing Biosynthesis Biotechnology Co., Ltd., Beijing, China), anti-HO-1 (1:200 dilution; cat. no. bs-2075R; Beijing Biosynthesis Biotechnology Co., Ltd.), primary mouse anti- $\beta$-actin (1:3,000 dilution; cat. no. sc-130300; Santa Cruz Biotechnology, Inc., Dallas, TX, USA) and anti- $\alpha$-tubulin (1:3,000 dilution; cat. no. 66031, ProteinTech Group, Inc.) overnight at $4^{\circ} \mathrm{C}$. The membrane was then incubated for an additional $2 \mathrm{~h}$ at room temperature with goat anti-mouse secondary antibody (1:4,000 dilution; cat. no. ZB-2305; Zhongshan Golden Bridge Biotechnology Co Ltd., Beijing, China) for $\beta$-actin and $\alpha$-tubulin, or goat anti-rabbit secondary antibody (1:2,000 dilution; cat. no. SA00001-2; ProteinTech 
Group, Inc.) for other proteins. The immunoreactive proteins were detected using an enhanced chemiluminescence western blotting detection kit (Thermo Fisher Scientific, Inc., Waltham, MA, USA). The relative protein expression was quantified with Molecular Dynamics Image Quant software (GE Healthcare Life Sciences, Chalfont, UK). Expression of nuclear Nrf2 was normalized to $\alpha$-tubulin, while expression of the other proteins was normalized to $\beta$-actin.

Measurement of GSH and oxidized glutathione (GSSG) in rat lungs. Lung tissues $(\mathrm{n}=6)$ were homogenized with $10 \mathrm{ml}$ ice-cold lysis buffer $(50 \mathrm{mM}$ phosphate buffer containing $1 \mathrm{mM}$ EDTA) per gram of tissue. The homogenate was centrifuged at $10,000 \times \mathrm{g}$ for $15 \mathrm{~min}$ at $4^{\circ} \mathrm{C}$, and then deproteinated with 1.25 M metaphosphoric acid (cat. no. 04103, Sigma-Aldrich; Merck KGaA) and stored at $-20^{\circ} \mathrm{C}$. Total glutathione and oxidized glutathione levels were determined using a glutathione assay kit (Cayman Chemical Company, Ann Arbor, MI, USA), according to the manufacturer's protocol.

Measurement of lung ROS levels. Samples of lung tissue from rats in each group $(n=6)$ were homogenized using a Polytron homogenizer (Kinematica, Lucerne, Switzerland) to extract protein. The homogenate was centrifuged at 15,000 x $g$ for $30 \mathrm{~min}$ at $4^{\circ} \mathrm{C}$ and the supernatant was collected and stored at $-80^{\circ} \mathrm{C}$ for enzyme-linked immunosorbent assay (ELISA). ROS concentration in the lung tissues was measured using a ROS ELISA kit (cat. no. MAB7475; R\&D Systems, Inc., Minneapolis, MN, USA) according to the manufacturer's protocol. The absorbance was detected using a Tecan Sunrise microplate reader (Tecan Group,Ltd., Männedorf, Switzerland) at a wave length of $450 \mathrm{~nm}$ and the corresponding concentration was determined from the standard curve.

Statistical analysis. All data are presented as mean \pm standard deviation. Statistical analysis was performed using one-way analysis of variance followed by the least significant difference (LSD) test with SPSS 13.0 software (SPSS, Inc., Chicago, IL, USA). Linear regression analysis was used to evaluate the correlation of lung ROS level with MA-induced lung injury. $\mathrm{P}<0.05$ was considered to indicate a statistically significant difference.

\section{Results}

Comparison of the survival rate of rats in different groups In the present experiment, 10 rats in different groups were administrated with $0.9 \%$ saline, $5 \mathrm{mg} / \mathrm{kg} \mathrm{MA}$ and $10 \mathrm{mg} / \mathrm{kg}$ MA, respectively. The survival rate was assessed at the end of every week. During the time of the model establishment, there was no mortality in the control group, but the survival rate reduced from $100 \%$ in the M5 group and $90 \%$ in the M10 group at the end of the 1st week, to $80 \%$ in the M5 group and $60 \%$ in the M10 group at the end of the last week. Compared with the control group, the survival rate was significantly reduced in the M10 group ( $\mathrm{P}<0.05$; Fig. 1). The survival curves were compared using the log-rank test.

Effect of MA on heart rate (HR) and peak blood flow velocity of pulmonary arterial valves $(P A V)$. Blood flow velocity alters
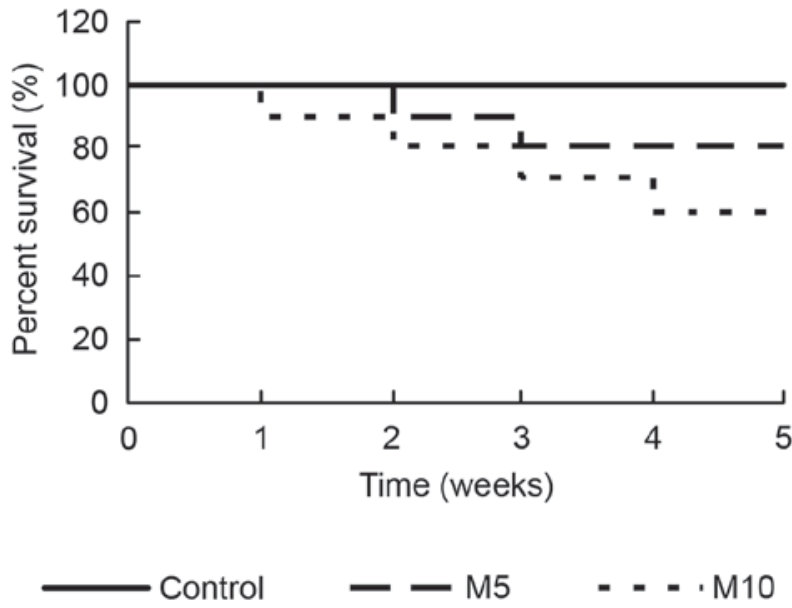

Figure 1. Comparison of survival rate of rats in different groups. Data are expressed as the mean \pm standard deviation. $\mathrm{M} 5,5 \mathrm{mg} / \mathrm{kg}$ methamphetamine; $\mathrm{M} 10,10 \mathrm{mg} / \mathrm{kg}$ methamphetamine.

with respiration (21). The fluctuation of respiratory blood flow velocity is helpful to evaluate the degree of damage of lung tissue in patients $(21,22)$. Ultrasonic imaging (Fig. 2A) revealed that $10 \mathrm{mg} / \mathrm{kg}$ MA significantly increased mean blood flow velocity (Fig. 2B) and HR (Fig. 2C) compared with the control.

HR was $\sim 330 \pm 60 \mathrm{bpm}$ in the control group, and following treatment with $5 \mathrm{mg} / \mathrm{kg}$ MA the.

PAV was $\sim 96 \pm 7.4 \mathrm{~cm} / \mathrm{s}$ in the control group, and following $5 \mathrm{mg} / \mathrm{kg}$ MA treatment, PAV did not significantly differ between the control group and M5 group ( $\mathrm{P}>0.05$; Fig. $2 \mathrm{~B}$ ). However, $10 \mathrm{mg} / \mathrm{kg}$ MA treatment significantly increased PAV to $136 \pm 14 \mathrm{~cm} / \mathrm{s}$ compared with the control group $(\mathrm{P}<0.01$; Fig. 2B).

HR was elevated to $360 \pm 120 \mathrm{bpm}$, but no significant differences were observed compared with the control group $(\mathrm{P}>0.05)$. However, $10 \mathrm{mg} / \mathrm{kg}$ MA treatment significantly increased HR to $540 \pm 180 \mathrm{bpm}$ compared with the control group $(\mathrm{P}<0.05$; Fig. 2C).

Histopathological analysis of lung injury induced by MA. To assess lung injury response to MA, histopathological analysis was performed on sections stained with hematoxylin and eosin. Following MA administration for 5 weeks, visible differences in the level of lung injury were observed in the M5 and M10 groups compared with the control group. Perivascular exudates, thickened alveolar septa, slight hemorrhage, airspace edema and inflammatory cell infiltration were observed in rats treated with MA (Fig. 3A). Only inflammatory cell infiltration was significantly increased in the M5 group compared with the control $(\mathrm{P}<0.05$; Fig. 3B). However, edema, hemorrhage, inflammatory cell infiltration and total lung injury scores were significantly increased in the M10 group compared with the control group $(\mathrm{P}<0.05, \mathrm{P}<0.05, \mathrm{P}<0.01$ and $\mathrm{P}<0.01$, respectively; Fig. $3 \mathrm{~B}$ and $\mathrm{C}$, respectively). Consistent with this injury pattern, the lung W/D weight ratios of rats were significantly increased in the M5 and M10 groups compared with the control $(\mathrm{P}<0.05$ and $\mathrm{P}<0.01$, respectively; Fig. 3D).

Effect of chronic MA exposure on Nrf2 expression in rat lungs. Cytoplasmic and nuclear extracts were subjected to 
A

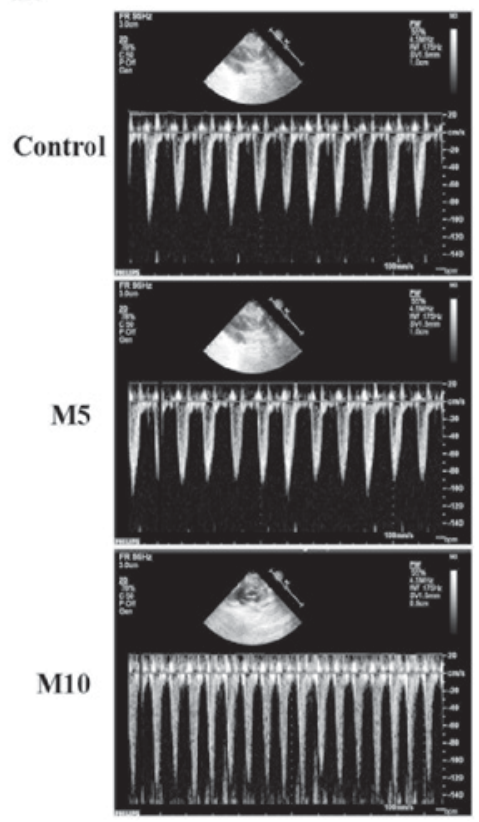

B

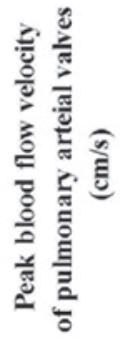

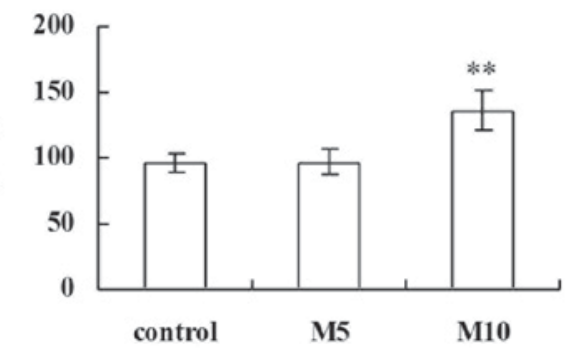

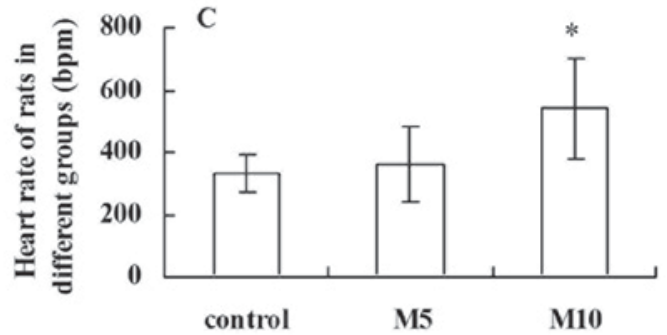

Figure 2. Doppler ultrasonic detection of physiological indexes. (A) Cardiovascular ultrasound imaging of rats in different groups. A frequency of $4.5 \mathrm{MHz}$ and one focal zone set at a depth of $0.5-2 \mathrm{~cm}$ were used to achieve detailed imaging. Comparison of the (B) heart rate and (C) peak blood flow velocity of pulmonary arterial valves in different groups. Data are expressed as the mean \pm standard deviation $(\mathrm{n}=6)$. ${ }^{*} \mathrm{P}<0.05,{ }^{* *} \mathrm{P}<0.01 \mathrm{vs}$. control group. M5, $5 \mathrm{mg} / \mathrm{kg}$ methamphetamine; M10, $10 \mathrm{mg} / \mathrm{kg}$ methamphetamine.

A

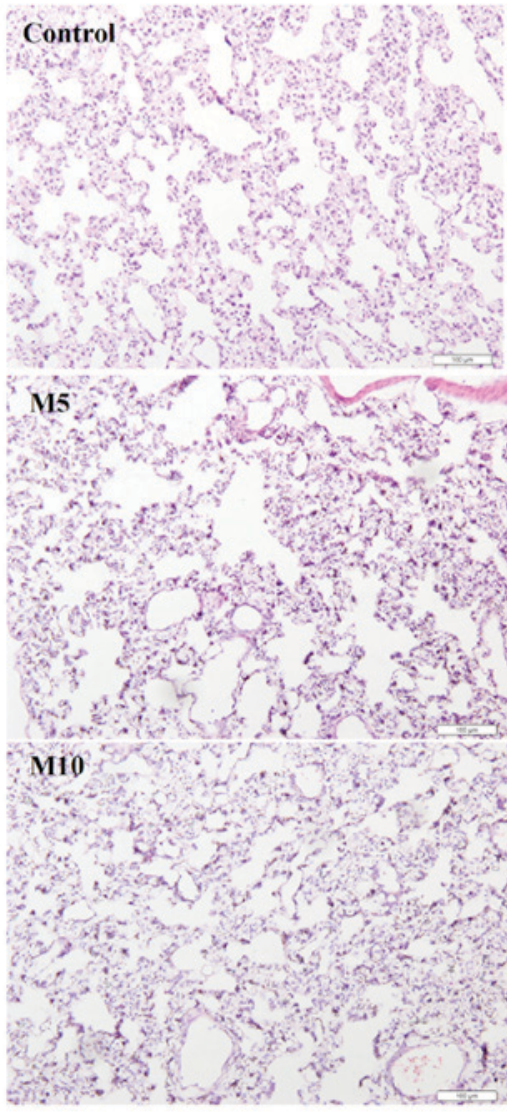

B
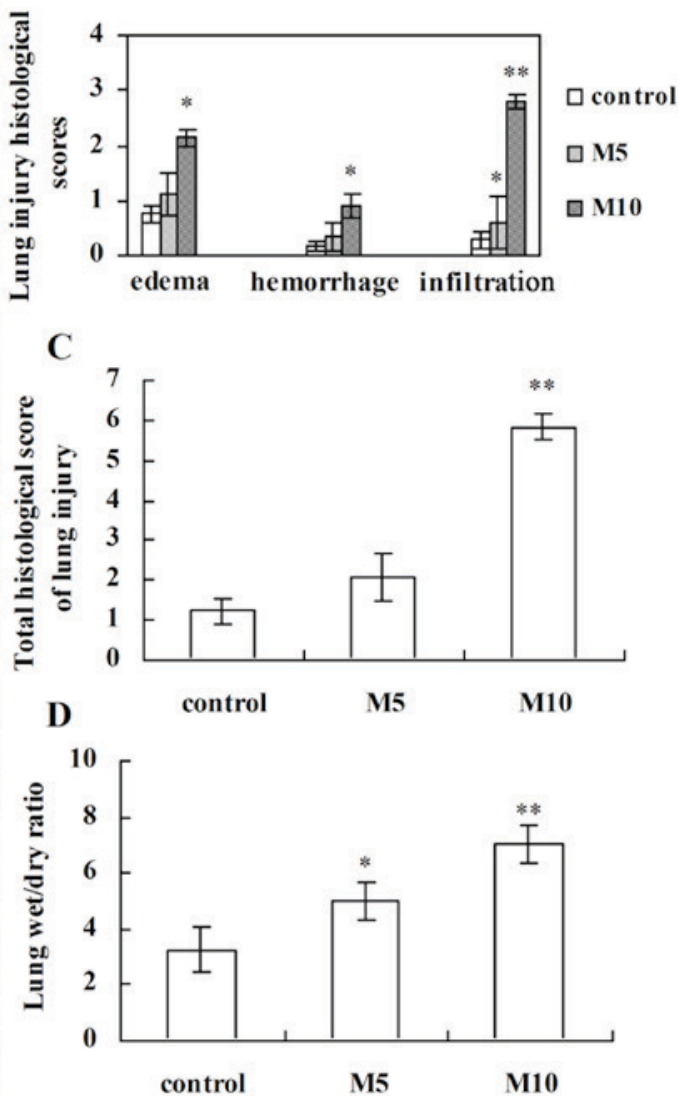

Figure 3. Histopathological analysis of lung injury induced by methamphetamine. (A) Representative images of lung injury following hematoxylin and eosin staining from the control, M5 and M10 groups (magnification, x200). (B) Histological scores for interstitial edema, hemorrhage and inflammatory cell infiltration. Each feature received a score of 0 , no injury; 1 , minimal injury; 2 , moderate injury or 3 , severe injury. (C) Total lung injury scores in different groups. The score for each rat was given as a minimum of 0 and a maximum of 9. (D) Comparison of lung wet-to-dry weight ratio in different groups. Data are expressed as the mean \pm standard deviation $(\mathrm{n}=6) .{ }^{*} \mathrm{P}<0.05,{ }^{* *} \mathrm{P}<0.01$ vs. control group. M5, $5 \mathrm{mg} / \mathrm{kg}$ methamphetamine; M10, $10 \mathrm{mg} / \mathrm{kg}$ methamphetamine. 
$\mathbf{A}$

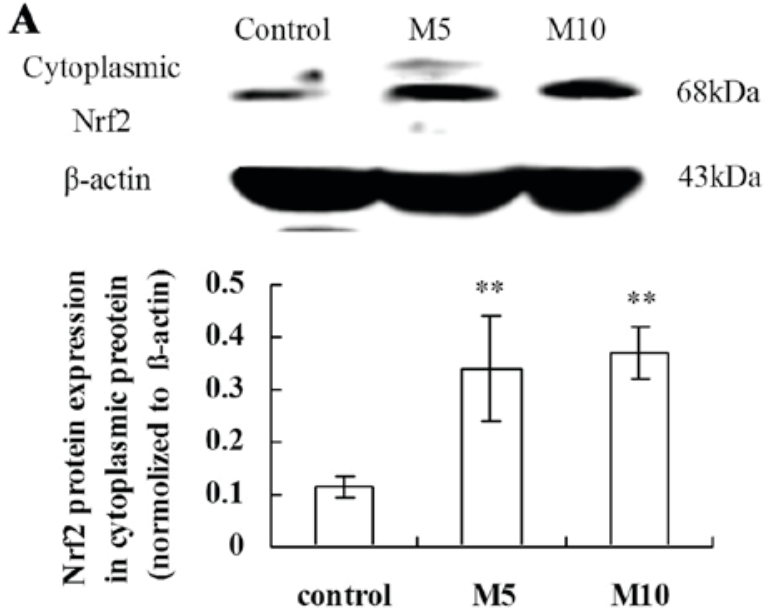

B
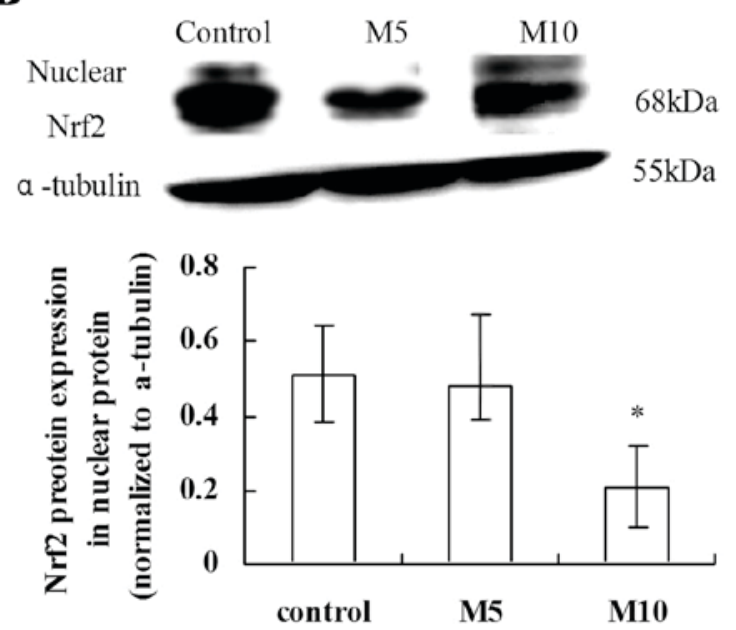

C

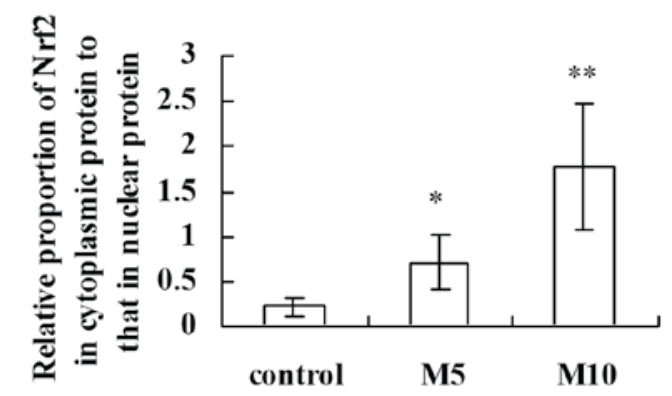

Figure 4. Effect of chronic exposure to methamphetamine on Nrf2 expression in rat lungs. (A) Expression of cytoplasmic Nrf2 protein in different groups. (B) Expression of nuclear Nrf2 protein in different groups. (C) Relative proportion of cytoplasmic Nrf2 protein and nuclear Nrf2 protein. Data are expressed the mean \pm standard deviation $(n=6) .{ }^{*} \mathrm{P}<0.05,{ }^{* *} \mathrm{P}<0.01$ vs. control group. Nrf2, nuclear factor erythroid-2-related factor $2 ; \mathrm{M} 5,5 \mathrm{mg} / \mathrm{kg}$ methamphetamine; M10, $10 \mathrm{mg} / \mathrm{kg}$ methamphetamine.

immunoblotting against Nrf2. MA significantly increased Nrf2 cytoplasmic protein expression levels in the M5 group compared with the control group $(\mathrm{P}<0.01$; Fig. $4 \mathrm{~A})$. Nrf2 nuclear protein expression levels were significantly reduced in the M10 group compared with the control group $(\mathrm{P}<0.05$; Fig. 4B). The relative proportion of Nrf2 cytoplasmic to nuclear protein was significantly increased in the M5 and M10 groups compared with the control group $(\mathrm{P}<0.05$ and $\mathrm{P}<0.01$, respectively; Fig. 4C). Therefore, MA treatment significantly
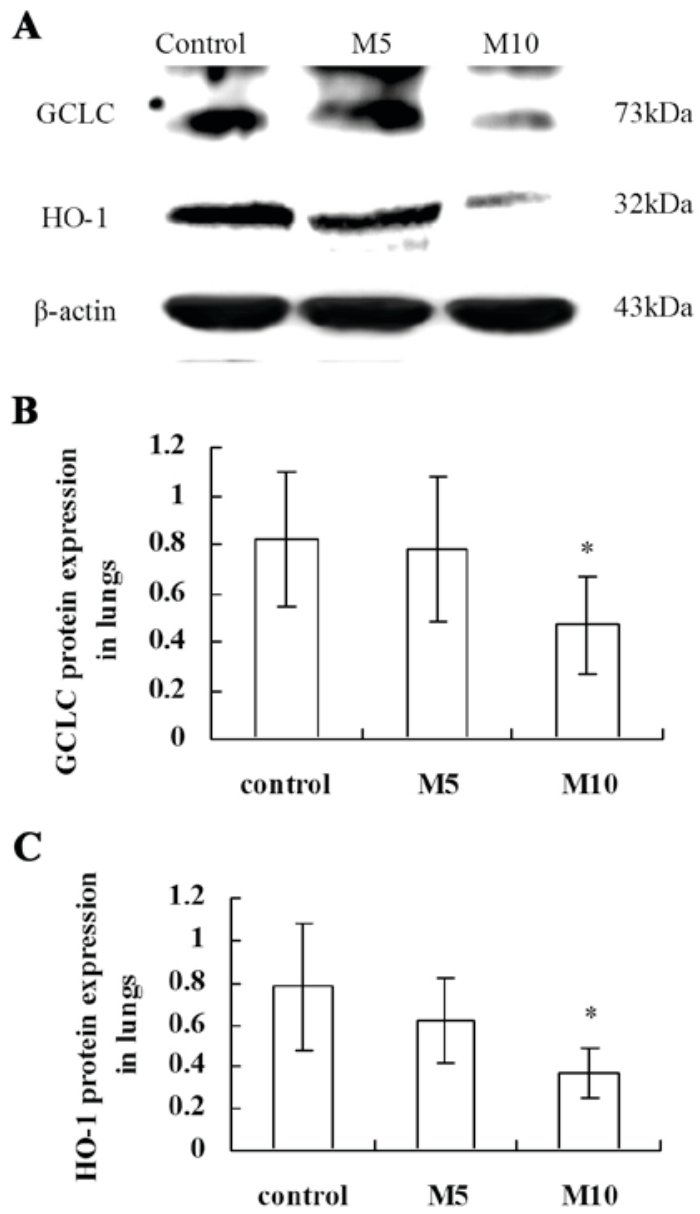

Figure 5. Effect of chronic exposure of MA on GCLC and HO-1 protein expression levels in rat lungs. (A) Representative western blot images and quantification of (B) GCLC and (C) HO-1 protein expression levels. Data are expressed as the mean \pm standard deviation $(n=6) .{ }^{*} \mathrm{P}<0.05$ vs. control group. MA, methamphetamine; GCLC, glutamate-cysteine ligase catalytic subunit C; HO-1, heme oxygenase-1; M5, $5 \mathrm{mg} / \mathrm{kg}$ methamphetamine; M10, $10 \mathrm{mg} / \mathrm{kg}$ methamphetamine.

suppressed the translocation of Nrf2 from the cytoplasm to nucleus and inhibited the Nrf2-mediated antioxidative defense in a concentration-dependent manner.

Inhibitory effect of MA on antioxidant enzymes GCLC and HO-1 in rat lungs. The western blot assay (Fig. 5A) demonstrated that the Nrf2 target genes GCLC $(\mathrm{P}<0.05$; Fig. 5B) and HO-1 $(\mathrm{P}<0.05$; Fig. 5C) protein expression levels were significantly reduced in the M10 group compared with the control group.

Effect of MA on GSH and GSSG protein expression levels in lung tissues. The GSH protein level and the ratio of GSH to GSSG (GSH/GSSG) are involved in antioxidative defense (23). In the present study, MA treatment significantly and dose-dependently downregulated lung GSH protein levels compared with the control (M5 group, $\mathrm{P}<0.05$; M10 group, $\mathrm{P}<0.01$; Fig. $6 \mathrm{~A}$ ), and lung GSSG protein levels were additionally significantly upregulated in the M10 group compared with the control group $(\mathrm{P}<0.01$; Fig. 6B). Associated with the decreased GSH protein level, the GSH/GSSG ratios were dose-dependently significantly reduced by MA treatment compared with the control 
$\mathbf{A}$

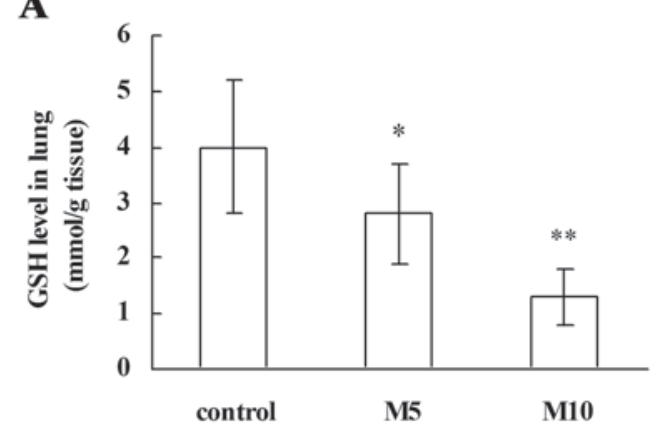

B
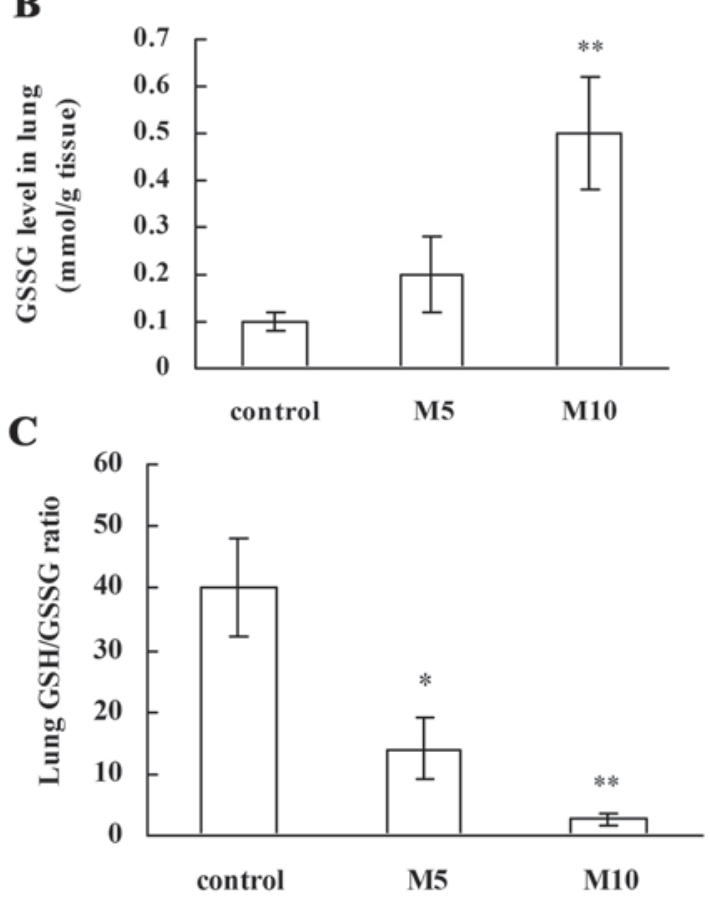

Figure 6. Effect of methamphetamine on GSH and GSSG in lung tissues. Protein expression levels of (A) GSH and (B) GSSG in rat lungs in different groups. (C) Comparison of the lung GSH/GSSG ratio in different groups. Data are expressed as the mean \pm standard deviation $(n=6) .{ }^{*} \mathrm{P}<0.05,{ }^{* *} \mathrm{P}<0.01$ vs. control group. GSH, glutathione; GSSG, oxidized glutathione; M5, $5 \mathrm{mg} / \mathrm{kg}$ methamphetamine; M10, $10 \mathrm{mg} / \mathrm{kg}$ methamphetamine.

(M5 group, $\mathrm{P}<0.05 ; \mathrm{M} 10$ group, $\mathrm{P}<0.01 ;$ Fig. 6C). These findings support that MA suppresses antioxidative defense in rat lungs.

Correlation between lung ROS levels and the indexes of lung injury. The results of ELISA revealed that, although no significant differences were observed in ROS level between the control and M5 groups, ROS levels were significantly increased in the M10 group compared with the control $(\mathrm{P}<0.01$; Fig. 7A). Linear regression analysis revealed a significant positive correlation between lung ROS levels and the indexes of lung injury: Lung injury scores $\left(\mathrm{R}^{2}=0.8,674 ; \mathrm{P}<0.05\right.$; Fig. $\left.7 \mathrm{~B}\right)$ and $\mathrm{W} / \mathrm{D}$ weight ratios $\left(\mathrm{R}^{2}=0.7,087 ; \mathrm{P}<0.05\right.$; Fig. $\left.7 \mathrm{C}\right)$. These results suggested that alterations in lung ROS levels coincided with alterations in lung injury induced by chronic exposure to MA.

\section{Discussion}

The results of the present study indicated that chronic exposure to MA induced an increase in HR and PAV and a decrease
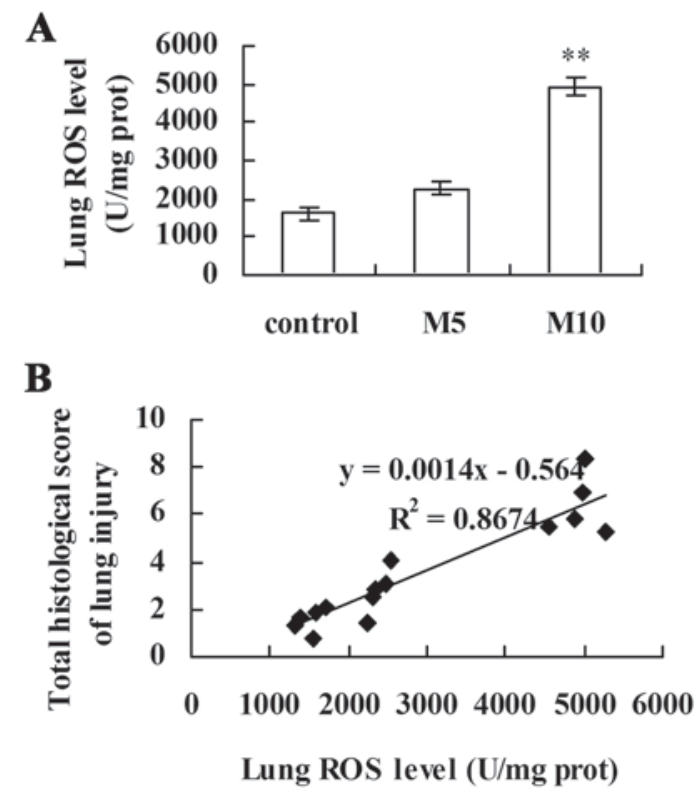

C

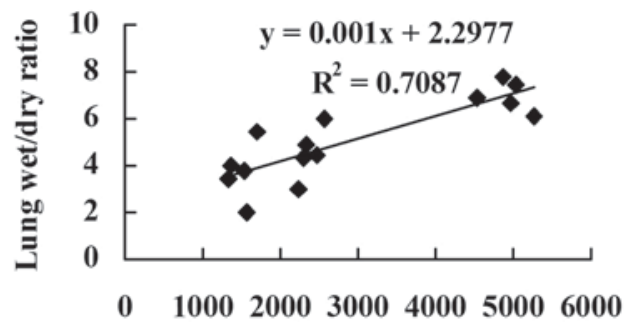

Lung ROS level (U/mg prot)

Figure 7. Correlation between lung ROS level and lung injury indexes. (A) Influence of methamphetamine on ROS level in rat lungs. Data are expressed as the mean \pm standard deviation $(\mathrm{n}=6) .{ }^{* *} \mathrm{P}<0.01 \mathrm{vs}$. control group. Correlation analysis between lung ROS levels and (B) total histological scores of lung injury $\left(\mathrm{R}^{2}=0.8674 ; \mathrm{P}<0.05\right)$ and $(\mathrm{C})$ lung wet/dry ratio $\left(\mathrm{R}^{2}=0.7087\right.$; $\mathrm{P}<0.05)$. ROS, reactive oxygen species; $\mathrm{M} 5,5 \mathrm{mg} / \mathrm{kg}$ methamphetamine; $\mathrm{M} 10,10 \mathrm{mg} / \mathrm{kg}$ methamphetamine.

in the survival rate of rats, and led to histopathological alterations common to lung injury: Perivascular exudates, airspace edema, slight hemorrhage and inflammatory cell infiltration. Furthermore, $10 \mathrm{mg} / \mathrm{kg}$ MA treatment significantly inhibited the expression of nuclear Nrf2 protein and Nrf2 target genes (GCLC and HO-1), and MA dose-dependently reduced GSH protein levels and the ratio of GSH/GSSG, accompanied by increases in ROS levels in rat lungs, which implied that MA suppresses Nrf2-mediated antioxidative properties. Linear regression analysis revealed that there was a positive correlation between lung ROS level and lung injury indexes, as determined by histological scores of lung injury and lung W/D ratio. These findings suggested that suppression of Nrf2-mediated antioxidative defense is associated with lung injury induced by chronic exposure to MA in rats.

The lungs are the principal organ exposed to MA abuse (24). Baylor et al (25) confirmed that inhalation of MA results in interstitial pulmonary fibrosis and progressive massive fibrosis in the absence of other causes. Previous studies have also demonstrated that chronic exposure to MA induces 
pulmonary toxicity, including pulmonary vascular remodeling and pulmonary inflammation $(10,19)$. Blood flow velocity changes with respiration. In the present study, $10 \mathrm{mg} / \mathrm{kg} \mathrm{MA}$ significantly increased the heart rate accompanied with the increasing peak blood flow velocity of pulmonary arterial valves, which reflected the clinical manifestation: palpitation and shortness of breath of pulmonary arterial hypertension (2). The fluctuation of blood flow velocity of pulmonary arterial valves is helpful to indirectly evaluate the degree of damage of lung tissue in patients $(21,22)$. In the present study, MA induced perivascular exudates, airspace edema, slight hemorrhage and inflammatory cell infiltration, which resulted in a decline in cardiopulmonary function and the survival rate of rats. These findings indicated that lung injury was one of the important causes of the mortality that resulted from chronic exposure to MA.

Oxidative stress is a general term used for describing pathologies of various diseases at a molecular level, and reflects an imbalance between free radical production and antioxidant defense of the biological system $(26,27)$. ROS activate redox-sensitive transcription factors including Nrf2 during oxidative stress $(11,28,29)$. Nrf2 is a key molecule in the antioxidant defense system (30). Nrf2 is a transcription factor responsible for regulating a group of antioxidant enzymes including GCLC and HO-1 against oxidative stress $(31,32)$. The present study demonstrated that MA increased cytoplasmic Nrf2 protein expression, but significantly inhibited nuclear Nrf2 protein expression levels. MA also significantly increased the relative proportion of cytoplasmic Nrf2 compared with nuclear Nrf2, which confirmed that MA significantly suppressed the translocation of Nrf2 from the cytoplasm to the nucleus. Furthermore, MA significantly inhibited the protein expression of Nrf2 target genes, the antioxidant enzymes GCLC and HO-1, in rat lungs. These findings suggested that chronic exposure to MA inhibits the Nrf2-mediated antioxidative defense system.

GSH is an abundant endogenous antioxidant and a critical regulator of oxidative stress and immune function $(33,34)$. Cellular GSH homeostasis is maintained by catalyzing the reduction of GSSG into GSH $(34,35)$. As a co-factor for various enzymatic reactions, GSH reduces cellular ROS levels to protect against oxidative stress-induced injury (35-37). ROS can be produced from multiple sources, including mechanical ventilation, surgical trauma, manipulated lung tissue, and hyperoxia in the ventilated lung (38). ROS have been demonstrated to regulate inflammation and structural changes in the lungs (39). In the present study, it was revealed that MA dose-dependently reduced the GSH level and the ratio of GSH/GSSG, accompanied by increases in the ROS level in rat lungs. In addition, there was a positive correlation between lung ROS levels and histological scores of lung injury and the lung W/D ratio, which suggested that alterations in lung ROS levels coincided with the degree of lung injury induced by chronic exposure to MA. Taken together, this indicated that the GSH-dependent Nrf2 antioxidant defense was involved in regulating oxidative stress-induced lung injury by MA.

In conclusion, suppression of Nrf2-mediated antioxidative defense was demonstrated to be associated with lung injury induced by chronic exposure to MA in rats. The present study suggests that Nrf2, as the key factor in the balance between oxidant load and antioxidative capacity, may be an important therapeutic target for MA-induced chronic lung toxicity.

\section{Acknowledgements}

The present study was funded by the National Natural Science Foundation of China (grant no. 81503058) and the Natural Science Foundation of Liaoning Province (grant no. 2014021065).

\section{References}

1. Patel D, Desai GM, Frases S, Cordero RJ, DeLeon-Rodriguez CM, Eugenin EA, Nosanchuk JD and Martinez LR: Methamphetamine enhances Cryptococcus neoformans pulmonary infection and dissemination to the brain. MBio 4: pii: e00400-13, 2013.

2. Rothman RB and Baumann MH: Methamphetamine and idiopathic pulmonary arterial hypertension: Role of the serotonin transporter. Chest 132: 1412-1413, 2007.

3. Volkow ND, Fowler JS, Wang GJ, Shumay E, Telang F, Thanos PK and Alexoff D: Distribution and pharmacokinetics of methamphetamine in the human body: Clinical implications. PLoS One 5: e15269, 2010.

4. Eba S, Hoshikawa Y, Moriguchi T, Mitsuishi Y, Satoh H, Ishida K, Watanabe T, Shimizu T, Shimokawa H, Okada Y, et al: The nuclear factor erythroid 2-related factor 2 activator oltipraz attenuates chronic hypoxia-induced cardiopulmonary alterations in mice. Am J Respir Cell Mol Biol 49: 324-333, 2013.

5. Van Houten B: Pulmonary arterial hypertension is associated with oxidative stress-induced genome instability. Am J Respir Crit Care Med 192: 129-130, 2015.

6. Ushio-Fukai M: Localizing NADPH oxidase-derived ROS. Sci STKE 2006: re8, 2006.

7. Auten RL and Davis JM: Oxygen toxicity and reactive oxygen species: The devil is in the details. Pediatr Res 66: 121-127, 2009.

8. Chiou SY, Lee YS, Jeng MJ, Tsao PC and Soong WJ: Moderate hypothermia attenuates oxidative stress injuries in alveolar epithelial A549 cells. Exp Lung Res 39: 217-228, 2013.

9. Huang $\mathrm{CH}$, Yang ML, Tsai CH, Li YC, Lin YJ and Kuan YH: Ginkgo biloba leaves extract (EGb 761) attenuates lipopolysaccharide-induced acute lung injury via inhibition of oxidative stress and NF- $\kappa \mathrm{B}$-dependent matrix metalloproteinase-9 pathway. Phytomedicine 20: 303-309, 2013.

10. Wang Y, Liu M, Wang HM, Bai Y, Zhang XH, Sun YX and Wang HL: Involvement of serotonin mechanism in methamphetamine-induced chronic pulmonary toxicity in rats. Hum Exp Toxicol 32: 736-746, 2013.

11. Montes S, Juárez-Rebollar D, Nava-Ruíz C, Sánchez-García A, Heras-Romero $\mathrm{Y}$, Rios $\mathrm{C}$ and Méndez-Armenta $\mathrm{M}$ : Immunohistochemical study of Nrf2-antioxidant response element as indicator of oxidative stress induced by cadmium in developing rats. Oxid Med Cell Longev 2015: 570650, 2015.

12. Komaravelli N and Casola A: Respiratory viral infections and subversion of cellular antioxidant defenses. J Pharmacogenomics Pharmacoproteomics 5: pii: 1000141, 2014

13. Kim SG, Lee WH and Kim YW: Nrf2 (NF-E2-Related Factor2). In: Encyclopedia of Signaling Molecules. Choi S (ed). 1st edition. Springer New York, NY, pp1262-1268, 2012.

14. Kim J, Cha YN and Surh YJ: A protective role of nuclear factor-erythroid 2-related factor-2 (Nrf2) in inflammatory disorders. Mutat Res 690: 12-23, 2010.

15. Atia AE and Abdullah AB: Modulation of Nrf2/Keap1 pathway by dietary phytochemicals. Int J Res Med Sci 2: 375-381, 2014

16. D'Oria V, Petrini S, Travaglini L, Priori C, Piermarini E, Petrillo S, Carletti B, Bertini E and Piemonte F: Frataxin deficiency leads to reduced expression and impaired translocation of NF-E2-related factor (Nrf2) in cultured motor neurons. Int J Mol Sci 14: 7853-7865, 2013.

17. Chiang HS and Maric M: Lysosomal thiol reductase negatively regulates autophagy by altering glutathione synthesis and oxidation. Free Radic Biol Med 51: 688-699, 2011. 
18. Howden R: Nrf2 and cardiovascular defense. Oxid Med Cell Longev 2013: 104308, 2013.

19. Liu M, Wang Y, Wang HM, Bai Y, Zhang XH, Sun YX and Wang HL: Fluoxetine attenuates chronic methamphetamine-induced pulmonary arterial remodelling: Possible involvement of serotonin transporter and serotonin 1B receptor. Basic Clin Pharmacol Toxicol 112: 77-82, 2013.

20. Zhao Z, Tang X, Zhao X, Zhang M, Zhang W, Hou S, Yuan W, Zhang H, Shi L, Jia H, et al: Tylvalosin exhibits anti-inflammatory property and attenuates acute lung injury in different models possibly through suppression of $\mathrm{NF}-\kappa \mathrm{B}$ activation. Biochem Pharmacol 90: 73-87, 2014.

21. Kudo K, Terae S, Ishii A, Omatsu T, Asano T, Tha KK and Miyasaka K: Physiologic change in flow velocity and direction of dural venous sinuses with respiration: MR venography and flow analysis. AJNR Am J Neuroradiol 25: 551-557, 2004

22. Halimi KE, Negadi M, Bouguetof H, Zemour L, Boumendil D and Chentouf Mentouri Z: Respiratory variations in aortic blood flow velocity and inferior vena cava diameter as predictors of fluid responsiveness in mechanically ventilated children using transthoracic echocardiography in a pediatric PICU. Critical Care 19 (Suppl 1): S181, 2015.

23. Qin T, Yin Y, Yu Q and Yang Q: Bursopentin (BP5) protects dendritic cells from lipopolysaccharide-induced oxidative stress for immunosuppression. PLoS One 10: e0117477, 2015.

24. Wells SM, Buford MC, Braseth SN, Hutchison JD and Holian A: Acute inhalation exposure to vaporized methamphetamine causes lung injury in mice. Inhal Toxicol 20 : 829-838, 2008.

25. Baylor PA, Sobenes JR and Vallyathan V: Interstitial pulmonary fibrosis and progressive massive fibrosis related to smoking methamphetamine with talc as filler. Respir Care 58: e53-e55, 2013.

26. Antus B, Drozdovszky O, Barta I and Kelemen K: Comparison of airway and systemic malondialdehyde levels for assessment of oxidative stress in cystic fibrosis. Lung 193: 597-604, 2015.

27. Sellamuthu PS, Arulselvan P, Kamalraj S, Fakurazi S and Kandasamy $\mathrm{M}$ : Protective nature of mangiferin on oxidative stress and antioxidant status in tissues of streptozotocin-induced diabetic rats. ISRN Pharmacol 2013: 750109, 2013.

28. Singh S, Vrishni S, Singh BK, Rahman I and Kakkar P: Nrf2-ARE stress response mechanism: A control point in oxidative stress-mediated dysfunctions and chronic inflammatory diseases. Free Radic Res 44: 1267-1288, 2010.
29. Kovac S, Angelova PR, Holmström KM, Zhang Y, Dinkova-Kostova AT and Abramov AY: Nrf2 regulates ROS production by mitochondria and NADPH oxidase. Biochim Biophys Acta 1850: 794-801, 2015.

30. Messier EM, Day BJ, Bahmed K, Kleeberger SR, Tuder RM, Bowler RP, Chu HW, Mason RJ and Kosmider B: N-acetylcysteine protects murine alveolar type II cells from cigarette smoke injury in a nuclear erythroid 2-related factor-2-independent manner. Am J Respir Cell Mol Biol 48: 559-567, 2013.

31. Masuda Y, Vaziri ND, Li S, Le A, Hajighasemi-Ossareh M, Robles L, Foster CE, Stamos MJ, Al-Abodullah I, Ricordi C and Ichii H: The effect of Nrf2 pathway activation on human pancreatic islet cells. PLoS One 10: e0131012. 2015.

32. Ishii T, Itoh K, Takahashi S, Sato H, Yanagawa T, Katoh Y, Bannai S and Yamamoto M: Transcription factor Nrf2 coordinately regulates a group of oxidative stress-inducible genes in macrophages. J Biol Chem 275: 16023-16029, 2000.

33. Richie JP Jr, Nichenametla S, Neidig W, Calcagnotto A, Haley JS, Schell TD and Muscat JE: Randomized controlled trial of oral glutathione supplementation on body stores of glutathione. Eur J Nutr 54: 251-263, 2015

34. Sinha-Hikim I, Shen R, Kovacheva E, Crum A, Vaziri ND and Norris KC: Inhibition of apoptotic signalling in spermine-treated vascular smooth muscle cells by a novel glutathione precursor. Cell Biol Int 34: 503-511, 2010

35. Kim SH, Smith AJ, Tan J, Shytle RD and Giunta B: MSM ameliorates HIV-1 Tat induced neuronal oxidative stress via rebalance of the glutathione cycle. Am J Transl Res 7: 328-338, 2015.

36. Yang SR, Park JR and Kang KS: Reactive oxygen species in mesenchymal stem cell aging: Implication to lung diseases. Oxid Med Cell Longev 2015: 486263, 2015.

37. Jayakumar S, Kunwar A, Sandur SK, Pandey BN and Chaubey RC: Differential response of DU145 and PC3 prostate cancer cells to ionizing radiation: Role of reactive oxygen species, GSH and Nrf2 in radiosensitivity. Biochim Biophys Acta 1840: 485-494, 2014.

38. Xia R, Xu J, Yin H, Wu H, Xia Z, Zhou D, Xia ZY, Zhang L, Li H and Xiao X: Intravenous infusion of dexmedetomidine combined isoflurane inhalation reduces oxidative stress and potentiates hypoxia pulmonary vasoconstriction during one-lung ventilation in patients. Mediators Inflamm 2015: 238041, 2015.

39. Yang X, Yao H, Chen Y, Sun L, Li Y, Ma X, Duan S, Li X, Xiang R, Han J and Duan Y: Inhibition of glutathione production induces macrophage CD36 expression and enhances cellular-oxidized low density lipoprotein (oxLDL) uptake. J Biol Chem 290: 21788-21799, 2015. 